\title{
Contaminant DNA in bacterial sequencing experiments is a major source of false genetic variability
}

\author{
Galo A. Goig ${ }^{1 *}\left(\mathbb{D}\right.$, Silvia Blanco², Alberto L. Garcia-Basteiro ${ }^{2,3}$ and Iñaki Comas ${ }^{1,4}$
}

\begin{abstract}
Background: Contaminant DNA is a well-known confounding factor in molecular biology and in genomic repositories. Strikingly, analysis workflows for whole-genome sequencing (WGS) data commonly do not account for errors potentially introduced by contamination, which could lead to the wrong assessment of allele frequency both in basic and clinical research.

Results: We used a taxonomic filter to remove contaminant reads from more than 4000 bacterial samples from 20 different studies and performed a comprehensive evaluation of the extent and impact of contaminant DNA in WGS. We found that contamination is pervasive and can introduce large biases in variant analysis. We showed that these biases can result in hundreds of false positive and negative SNPs, even for samples with slight contamination. Studies investigating complex biological traits from sequencing data can be completely biased if contamination is neglected during the bioinformatic analysis, and we demonstrate that removing contaminant reads with a taxonomic classifier permits more accurate variant calling. We used both real and simulated data to evaluate and implement reliable, contamination-aware analysis pipelines.
\end{abstract}

Conclusion: As sequencing technologies consolidate as precision tools that are increasingly adopted in the research and clinical context, our results urge for the implementation of contamination-aware analysis pipelines. Taxonomic classifiers are a powerful tool to implement such pipelines.

\section{Background}

Whole-genome sequencing (WGS) has enhanced the study of complex biological phenomena in bacteria, such as population dynamics, host adaptation, or outbreaks of microbial infections $[1,2]$. In addition, democratization of high-throughput sequencing technologies and continuous improvements in laboratory procedures are also turning WGS into a promising alternative for the clinical diagnosis and surveillance of several pathogenic species [3-5]. Thus, many efforts in the basic and clinical research fields are directed to the improvement of bioinformatic pipelines to ensure the robustness of the conclusions drawn.

Central to many bacterial WGS bioinformatic pipelines is the identification of genetic variants. Incorrect identification of variants can have a major impact on several areas of

\footnotetext{
* Correspondence: ggoig@ibv.csic.es

${ }^{1}$ Institute of Biomedicine of Valencia, IBV-CSIC, St. Jaume Roig 11, 46010

Valencia, Spain

Full list of author information is available at the end of the article
}

microbiological research. Applications based on variant analysis include, but are not limited to, phylogenetics [6], phylodynamics and dating [7], genome-wide association studies [8], experimental evolution [9], epidemiological analyses [10], and drug development [11]. Furthermore, the frequency at which each variant is observed in a sample can be used to characterize population genetics processes. Analysis of the allele frequency spectrum enables the study of population dynamics of diversity within a host or coexistence of mixed lineages [12]. In the clinical field, variant analysis at a genomic scale allows the identification of pathogen species and genotypes, distinguish between relapse and superinfections, or prediction of resistance phenotypes and transmission links.

While many factors are taken into account when developing SNP calling pipelines, surprisingly, the potential role of contamination is seldomly considered [13]. However, misinterpretation of contaminated data can lead to draw incorrect conclusions about biological phenomena [14, 15]. 
Genomic databases are known to encompass contaminated sequences, with assembled genomes that can contain large genomic regions from non-target organisms $[16,17]$. Strikingly, a recent study revealed that deposited bacterial and archaeal assemblies are contaminated by human sequences that created thousands of spurious proteins [18]. While the potential impact of contaminants has been considered in fields like metagenomics or transcriptomics, most bacterial WGS analysis pipelines lack specific steps aimed to deal with contaminant data. This situation likely originates from the assumptions that microbiological cultures are mostly free of non-target organisms and that even if present, contaminant sequences are unlikely to map to the reference genomes or are removed using standard filter cutoffs. To date, the extent of contamination and its impact in bacterial re-sequencing pipelines has not been comprehensively assessed.

In this work, we use both real and simulated data to perform a detailed comparison of a standard bacterial mapping and SNP calling pipeline against 2 alternative contamination-aware approaches. First, we implement a taxonomic filter removing contaminant reads that allowed us to assess the extent of contamination and estimate its impact in a dataset comprising 2600 samples of 13 different species from 12 bacterial WGS projects. Second, we compare the performance of this taxonomic filter with a filter based on the similarity of the alignments and evaluate the impact of contamination in 8 WGS projects comprising 1500 samples of Mycobacterium tuberculosis (MTB) WGS samples.

We found that contamination events are frequent across bacterial WGS studies and can introduce large biases in variant analysis despite the use of stringent mapping and variant calling cutoffs. Importantly, this is not only true for culture-free sequencing strategies, but also for experiments sequencing from pure cultures. We show that the effect size is not dependent on the amount of contamination and that samples with even low-level contamination can accumulate dozens of errors, particularly for non-fixed SNPs. We demonstrate that removing contaminant reads with a taxonomic classifier allows the implementation of more accurate variant calling pipelines, and provide a validated workflow for WGS analysis of MTB.

\section{Results}

Contamination is common across WGS studies, even when sequencing from pure cultures

To assess the extent of contamination across bacterial WGS studies, we taxonomically classified the sequencing reads of 4194 WGS samples from 20 different studies using Kraken, a metagenomic read classifier that has been extensively used and evaluated in the literature. Out of these, 1553 samples corresponded to M. tuberculosis sequencing projects, here referred as the MTB dataset, and 2641 to 13 other bacterial species, here referred as the bacterial dataset (Table 1). According to taxonomic classifications, varying levels of contamination with non-target reads can be found in the different studies (Fig. 1). From the bacterial dataset, Legionella pneumophila, Acinetobacter baumannii, Listeria monocytogenes, Pseudomonas aeruginosa, and Neisseria gonorrhoeae studies showed the expected taxonomic profile from pure culture sequencing, since virtually all the reads are classified in their respective target genus. By contrast, contamination can be clearly found in the rest of studies from this dataset, with an average of $45 \%$ of samples per study having less than the $90 \%$ of the reads coming from the target organism. The Treponema pallidum study represents an extreme case, with its samples having an average of only $40 \%$ of reads coming from this organism. This result is expected since in this study the samples were sequenced directly from clinical specimens using a bait capture strategy. However, high levels of contamination can be found in other studies where sequencing is performed from pure cultures (Fig. 1a).

When looking at the MTB dataset, we also observed contamination to be common across studies (Fig. 1b). As expected, direct sequencing from clinical specimens and early positive mycobacterial growth indicator tubes (MGIT), which are inoculated with primary clinical samples, present higher levels of contamination in terms of both the number of samples contaminated and the proportion of non-target reads within them. Common contaminants for these samples comprise human DNA, and bacteria usually found in oral and respiratory cavities like Pseudomonas, Rothia, Streptococcus, or Actinomyces, and can constitute virtually all reads in some samples. However, as observed for the bacterial dataset, contamination was also detected in studies in which the sequenced DNA came from pure culture isolates. For instance, Bacillus, Negativicoccus, and Enterococcus represented up to $68 \%, 58 \%$, and $32 \%$, respectively, of different samples from the KwaZulu study. Strikingly, 17 out of 73 MTB samples from the Nigeria study were identified as Staphylococcus aureus (92 to 99\% of reads). The high-depth dataset was mostly free of contamination, with the exception of two samples for which $3.32 \%$ of $A$. baumannii and $2.83 \%$ of non-tuberculous mycobacteria (NTM) were identified (representing 795,887 and 920, 379 reads, respectively).

\section{A taxonomic filter to selectively analyze non-contaminant reads}

To assess the impact of these contamination events in bacterial WGS analysis, we compared the outcomes in 
Table 1 Studies analyzed

\begin{tabular}{|c|c|c|c|c|}
\hline Study name & Publication & Runs analyzed & Sample source & Dataset \\
\hline Mozambique & Unpublished & 138 & Clinical isolates & MTB dataset \\
\hline Kwazulu-Natal & Cohen et al. 2015 & 433 & Single colonies from clinical isolates & MTB dataset \\
\hline Nigeria & Senghore et al. 2017 & 73 & Clinical isolates & MTB dataset \\
\hline Belarus & Wollenberg et al. 2017 & 552 & Clinical isolates & MTB dataset \\
\hline High-depth sequencing & Trauner et al. 2017 & 63 & Clinical isolates & MTB dataset \\
\hline Sputum-capture sequencing & Brown et al. 2015 & 58 & $\begin{array}{l}\text { Clinical respiratory specimens (culture-free sequencing } \\
\text { with a bait capture strategy) }\end{array}$ & MTB dataset \\
\hline Sputum-direct sequencing & Votintseva et al. 2017 & 68 & $\begin{array}{l}\text { Clinical respiratory specimens (direct culture-free } \\
\text { sequencing) }\end{array}$ & MTB dataset \\
\hline MGIT sequencing* & Pankhurst et al. 2016 & 168 & Early-positive MGIT cultures (liquid) & MTB dataset \\
\hline A. baumannii & Willems et al. 2016 & 36 & Single-colony recultured in broth & Bacterial dataset \\
\hline C. difficile & Stone et al. 2016 & 54 & Pooled single-colony isolates & Bacterial dataset \\
\hline Enterococcus $^{\dagger}$ & Tyson et al. 2018 & 197 & Isolates from retail meats & Bacterial dataset \\
\hline K. pneumoniae & Holt et al. 2015 & 285 & Human, animal, and environmental isolates & Bacterial dataset \\
\hline L. monocytogenes & Halbedel et al. 2018 & 424 & Clinical isolates from human & Bacterial dataset \\
\hline L. pneumophila & Timms et al. 2018 & 48 & Pure culture isolates from human and cooling towers & Bacterial dataset \\
\hline N. gonorrhoeae & Yahara et al. 2018 & 272 & Pure culture isolates from human & Bacterial dataset \\
\hline$P$. aeruginosa & Marvig et al. 2015 & 445 & Clinical isolates from human & Bacterial dataset \\
\hline S. aureus & Aanensen et al. 2016 & 337 & Clinical isolates from 186 hospitals in 21 countries & Bacterial dataset \\
\hline S. enterica & Gymoese et al. 2017 & 366 & Human, animal, and environmental isolates & Bacterial dataset \\
\hline T. pallidum & Pinto et al. 2016 & 25 & $\begin{array}{l}\text { Clinical specimens (culture-free sequencing with a bait } \\
\text { capture strategy) }\end{array}$ & Bacterial dataset \\
\hline Vibrio $^{\ddagger}$ & Greig et al. 2018 & 152 & Clinical isolates from human & Bacterial dataset \\
\hline
\end{tabular}

*This study included sequencing samples from non-MTB organisms. We analyzed the 168 reported as MTB by the authors

${ }^{+}$This study included sequencing samples from 2 species ( $E$. faecalis and E. faecium)

${ }^{\ddagger}$ This study included sequencing samples from different Vibrio species. We only analyzed the 152 reported as $V$. cholerae by the authors

variant calling for each sample before and after removing contaminant reads as classified by Kraken. We refer to this contamination removal methodology as a "taxonomic filter" (detailed in the "Material and methods" section). To assess whether our Kraken setup can be safely used to remove contaminant reads across the analyzed datasets, we first estimated the proportion of reads that can be classified up to the level of species and genus for each organism using simulated sequence reads from the corresponding reference genome. This analysis was performed both including and excluding the reference genomes from the database (Additional file 1: Table S1; Additional file 2: Table S2). For most of the organisms, more than the $99 \%$ of the reads could be classified at species level for $250 \mathrm{bp}$ simulated Illumina MiSeq reads (median $=99.35 \%$; 99.07\% excluding the reference) with the exceptions of $K$. pneumoniae $(97.86 \%$; $97.86 \%$ excluding the reference), S. aureus (95.01\%; 94.98\% excluding the reference), and T. pallidum (93.54\%; 92.96\% excluding the reference). Additionally, excluding the reference genome caused Enterococcus faecalis to drop from 99.55 to $90.59 \%$. For $100 \mathrm{bp}$ simulated Illumina HiSeq reads, the proportion of reads classified for each organism was lower in every case (median $=98.79 \%$; 97.96\% excluding the reference) with a dramatic drop for T. pallidum $(72.74 \%$; $71.25 \%$ excluding the reference), and with the exception of $M$. tuberculosis that remained at 99.98\%. At genus level, Kraken was able to classify most of the reads of each organism (median $=99.89 \%$ and $99.61 \%$ excluding the reference for $250 \mathrm{bp}$ simulations; median $=99.77 \%$ and $99.1 \%$ excluding the reference for $100 \mathrm{bp}$ simulations) with the exception of $S$. aureus that remained around $95 \%$ in every case and E. faecalis that was $91.17 \%$ after excluding the reference genome. Interestingly, for $T$. pallidum, which showed to be the most difficult organism to classify at species level, $100 \%$ of reads were classified at genus level even after excluding the reference genome. Therefore, to safely analyze the effect that contaminant reads have in WGS of the bacterial dataset, we applied the Kraken-based taxonomic filter at the genus level (e.g., we removed all non-Acinetobacter reads from the A. baumannii study).

Second, we scanned all the WGS samples to estimate the maximum proportion of reads Kraken is capable of classify as the target organism in real samples 


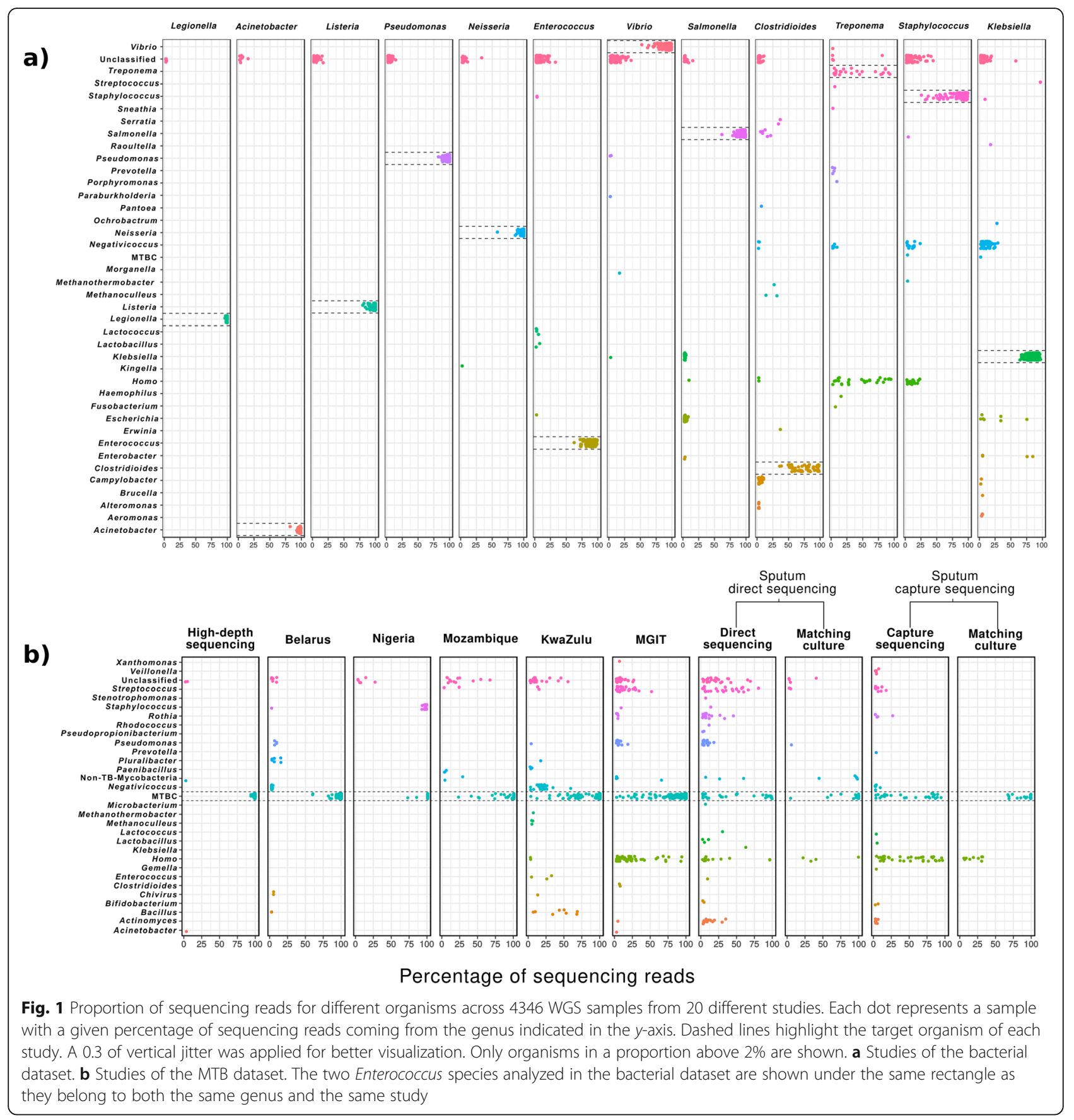

(Additional file 1: Table S1). In most cases, there was at least one sample per bacteria that could be classified as well as the reference genome (median difference between real and simulated sequencing of $1 \%$ at species level and $0.35 \%$ at genus level). The higher difference was observed for $T$. pallidum for which the maximum number of reads classified in a real WGS sample at genus level was $94.75 \%$. This most likely reflects that sequencing directly from clinical specimens usually produces contaminated samples.
Third, a fraction of reads that actually come from the target organism may be misclassified, and thus, such reads would be incorrectly removed by the taxonomic filter. In order to estimate the magnitude of this error in our analysis, we used Bracken to calculate the fraction of misclassified reads that were expected to actually belong to the target organism. Overall, the proportion of reads incorrectly eliminated by the taxonomic filter as estimated by Bracken was very low (median $=0.11 \%$, IQR $=1.32 \%$. This proportion varied 
between different organisms (Additional file 3: Table S3). For example, whereas for several organisms like $A$. baumannii, only $0.07 \%$ of the reads eliminated by the taxonomic filter are estimated to actually belong to Acinetobacter, in the case of $K$. pneumoniae, in which a high proportion of reads remain unclassified or are assigned to Negativicoccus massiliensis, 3.65\% are estimated to actually belong to Klebsiella.

Additionally, it can be argued that highly contaminated samples are likely to be detected by basic sequencing quality controls regardless of the implementation of specific contamination-control analysis. In contrast, contaminating reads would likely pass unnoticed when enough data from the target organism is produced and quality parameters like sequencing depth or genome coverage meet certain criteria. Furthermore, the measures of the impact of contamination in SNP analysis may be biased by including highly contaminated data. Thus, for the following analyses, we discarded samples with contamination higher than $50 \%$ or depths lower than $40 \times$ (only a minimum of $20 \times$ was required for $T$. pallidum, see the "Material and methods" section for a further explanation). From the initial 2641 samples of the bacterial dataset, 2233 met these criteria (408 had less than $40 \times$ depth and 16 had more than $50 \%$ of contamination (Additional file 4: Table S4)).

\section{Contamination impacts bacterial WGS analysis}

The expected effect of mapped contaminant reads is to produce mixed calls, leading to the identification of false positive variable SNPs (vSNPs). These false positive calls would alter the frequencies calculated at a given position, which might also produce false negative fixed SNPs (fSNPs) by lowering the frequency below the required cutoff to call fixed variants $(90 \%$ frequency in this work). The Pearson correlation coefficient between removing vSNPs and recovering fSNPs was of 0.76 (Fig. 2). However, not all the contaminant reads are expected to affect positions with fSNPs, and in fact, for 405 samples (18\%), the taxonomic filter removed the false positive vSNPs without affecting any fSNP. Similarly, in 38 samples (3\%), we observed the recovery of at least 1 false negative fSNP without removal of vSNPs. Notably, we did not observe a correlation between the number of vSNPs removed and the degree of contamination of a sample (Pearson's correlation coefficient $=-0.06$ ) (Table 2). This result suggests that the impact in variant analysis is highly dependent on the identity of both the contaminant and the target organisms, rather than the number of contaminating reads.

Overall, the impact of removing contaminant reads on vSNP and fSNP inference depended heavily on the species considered. For example, virtually, no change was observed for N. gonorrhoeae samples (Table 2, Fig. 3) while a mean number of 89 vSNPs were removed and 57 fSNPs recovered for A. baumannii samples. The greatest change was observed for K. pneumoniae, S. aureus, and Salmonella enterica datasets. However, in these cases, the impact of contaminant reads might be overestimated due to the incorrect elimination of target reads by the taxonomic filter. In many WGS applications, genetic variants are not analyzed on a sample basis but across the entire dataset. We therefore evaluated the impact of contaminant reads on polymorphic positions called across datasets. On average, the total number of polymorphic positions was reduced by $1.51 \%$ for fSNPs (range 0-6\%) and 8.67\% for vSNPs (range 0-41\%) (Fig. 3, Additional file 5: Table S5).

We also observed a small proportion of fSNPs to be systematically removed by the taxonomic filter (median $=0.2 \%$ of fSNPs, ranging from 0 to $5.6 \%$ between studies; Additional file 6: Table S6). Those positions can be considered false negatives introduced by the pipeline, including inconsistencies of the mapping software, and the inability of Kraken to classify a small proportion of reads disregarding their similarity to the reference genome. We observed this to occur mostly in regions of low coverage that could be the result of, for instance, hardto-map regions like repetitive elements or different strains coexisting in the same sample one of which has a deletion. In such regions, eliminating only one read can lead to greater differences in frequencies making the position fall below the required thresholds to call a fSNP (Additional file 7: Figure S1). Most of the fSNPs incorrectly removed after the taxonomic filter were caused by the inability of Kraken to classify some reads up to the level of genus. This behavior is a known limitation of the taxonomic classifiers for conserved regions among bacteria. However, in many cases, the incorrectly eliminated sequences corresponded to reads mapped with 100\% identity to the reference genome and surrounded by other sequences that were classified, even at the level of species, despite having several SNPs. This is probably due to the fact that for some reads, the $k$-mers in which they are decomposed do not allow Kraken to classify beyond a given taxonomic level, disregarding the sequence diversity. However, since the immediate contiguous reads can be correctly classified, this bias is normally compensated by the sequencing depth.

Unexpectedly, we also observed an inconsistency of the mapping software (bwa-mem) to be responsible for a small fraction of the SNPs that are either removed or recovered after applying the taxonomic filter. In these cases, we observed that the number of supporting reads at a given position differed in one read despite the fact that none of the reads mapping to that position were classified as contaminant. Surprisingly, we observed this to be the result of the exact same read, mapping to the 


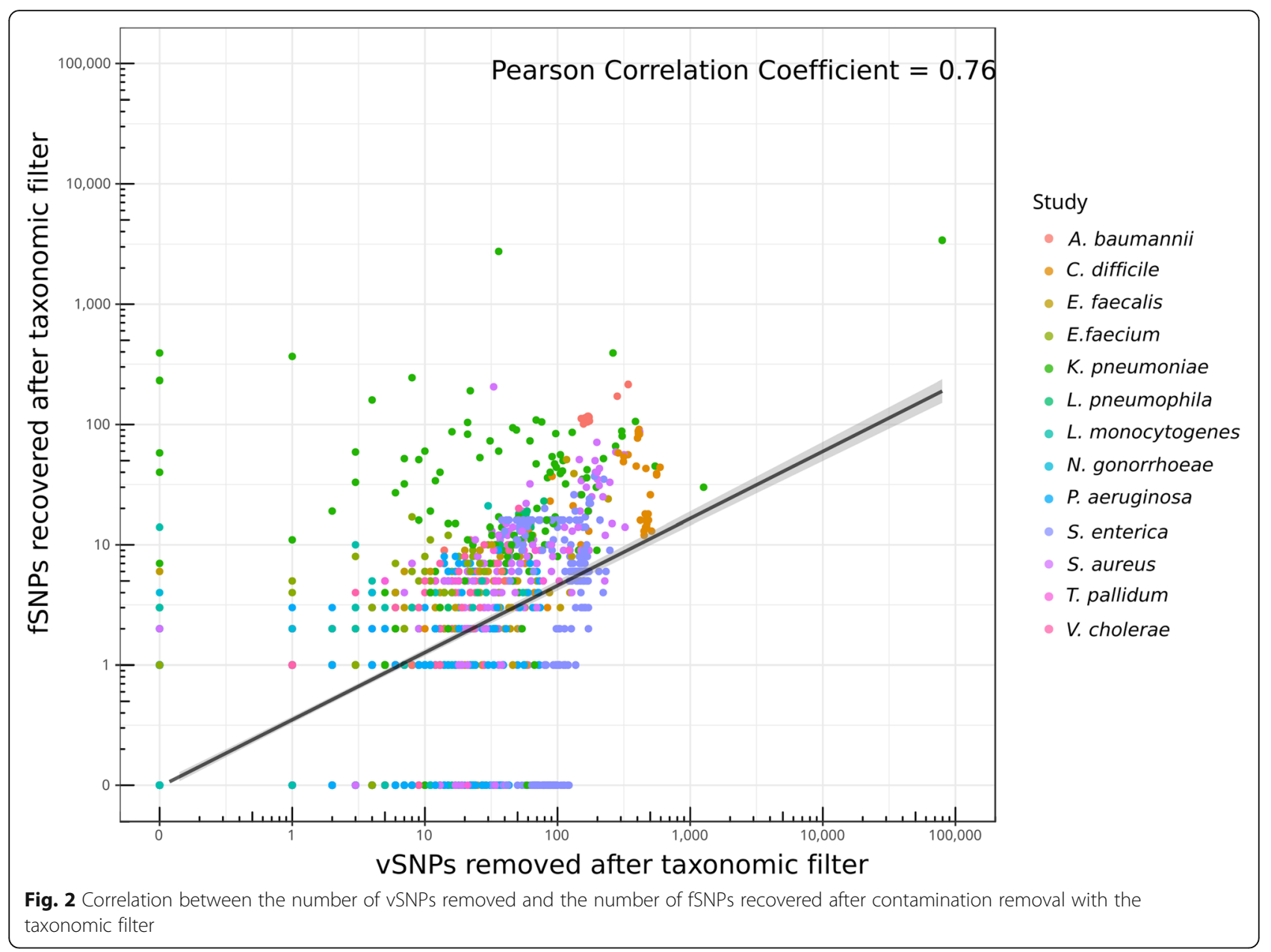

Table 2 Effect of applying the taxonomic filter in the variant analysis of samples of the bacterial dataset

\begin{tabular}{llllll}
\hline Study & $\begin{array}{l}\text { Mean percentage of } \\
\text { target organism (\%) }\end{array}$ & $\begin{array}{l}\text { Mean number of } \\
\text { vSNPs removed } \\
\text { (median; IQR) }\end{array}$ & $\begin{array}{l}\text { Mean number of } \\
\text { fSNPs recovered } \\
\text { (median; IQR) }\end{array}$ & $\begin{array}{l}\text { Pearson's correlation coefficient } \\
\text { between removal of vSNPs and } \\
\text { recovery of fSNPs }\end{array}$ & $\begin{array}{l}\text { Pearson's correlation coefficient } \\
\text { between removal of vSNPs and } \\
\text { percentage of target organism }\end{array}$ \\
\hline A. baumannii & 97.30 & $89(43 ; 165)$ & $57(10 ; 113)$ & 0.99 & 0.25 \\
C. difficile & 76.74 & $299(397 ; 379)$ & $27(16 ; 32)$ & 0.45 & 0.23 \\
E. faecalis & 89.96 & $30(19 ; 33)$ & $4(3 ; 5)$ & 0.65 & -0.13 \\
E. faecium & 94.38 & $9(5 ; 10)$ & $3(2 ; 5)$ & 0.47 & -0.45 \\
K. pneumoniae & 84.38 & $549(62 ; 112)$ & $73(13 ; 41)$ & 0.76 & -0.44 \\
L. pneumophila & 99.06 & $12(0 ; 8)$ & $3(0 ; 1)$ & 0.99 & -0.63 \\
L. monocytogenes & 98.42 & $2(0 ; 1)$ & $0(0 ; 0)$ & 0.49 & -0.43 \\
N. gonorrhoeae & 99.17 & $0(0 ; 0)$ & $0(0 ; 0)$ & 0.34 & -0.09 \\
P. aeruginosa & 97.43 & $9(2 ; 14)$ & $1(0 ; 1)$ & 0.50 & -0.11 \\
S. enterica & 95.01 & $97(91 ; 87)$ & $7(6 ; 12)$ & 0.14 & 0.02 \\
S. aureus & 91.42 & $50(22 ; 50)$ & $9(3 ; 9)$ & 0.54 & -0.10 \\
T. pallidum & 39.75 & $45(34 ; 52)$ & $6(5 ; 4)$ & 0.63 & -0.48 \\
V. cholerae & 91.32 & $9(5 ; 16)$ & $2(1 ; 3)$ & 0.76 & -0.56 \\
\hline
\end{tabular}




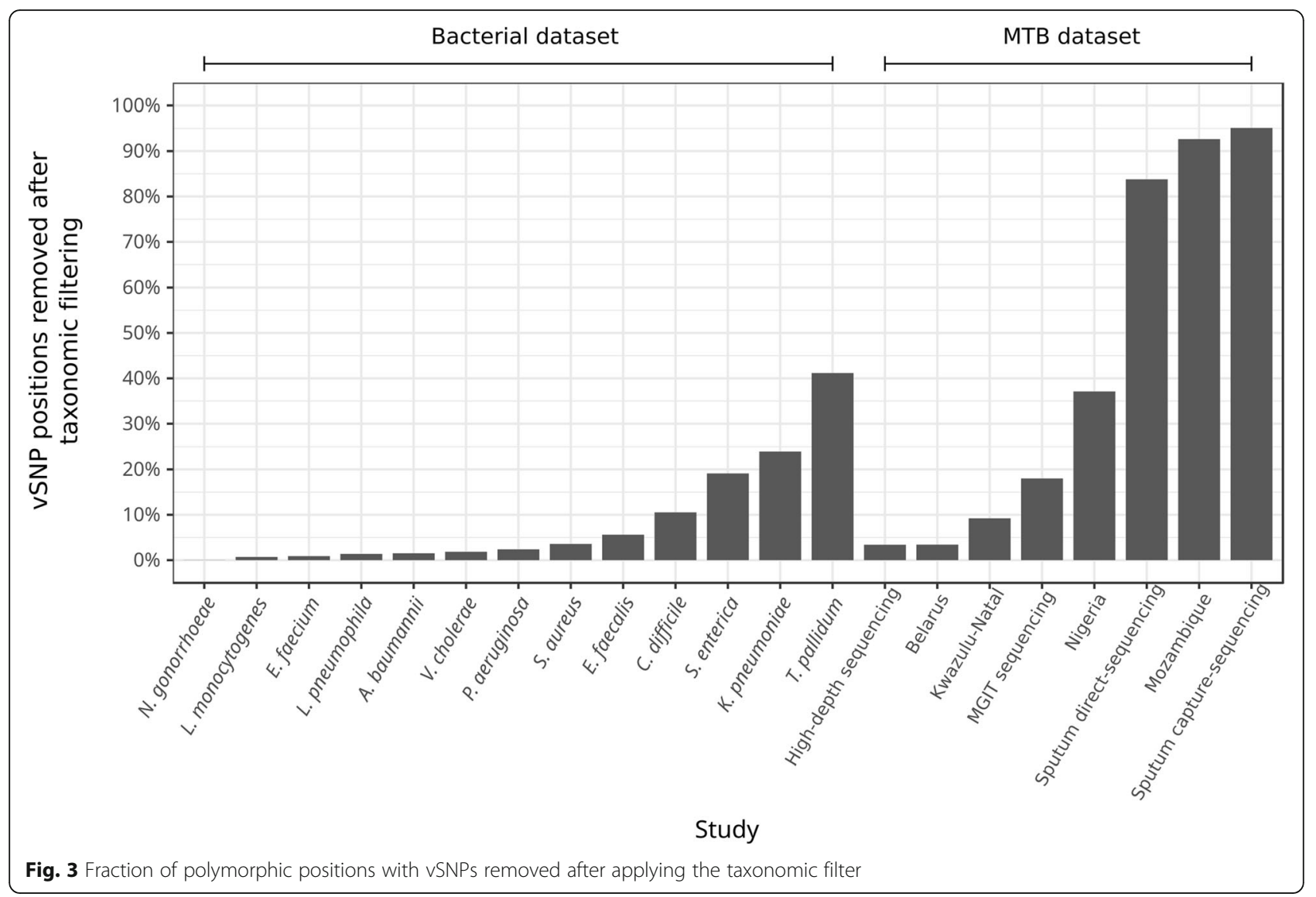

same genomic position, but with different qualities for the filtered and non-filtered fastq files. The fact that the fastq files are different (because some reads are removed in the filtered fastq) makes bwa to produce different results for a small number of reads (between 1 and 6 in our tests) that are filtered because of the mapping quality cutoff (60). We confirmed this behavior by randomly sorting and mapping different times a set of fastq files (tested versions 7.10, 7.12, and 7.17). This is likely due to the heuristics implemented in the seed-and-extension algorithm of bwa-mem.

Interestingly, we observed Negativicoccus massiliensis to be present at high proportions in several datasets. Analyzing a subset of these reads using the NCBI blast utility revealed that they present nucleotide similarity with eukaryotic organisms (e.g., Cyprinus carpio and Plasmodium vivax). Despite being contaminant reads, their classification as N. massiliensis is clearly artifactual, probably due to the absence of eukaryotic organisms in our database other than human. Similarly, Kraken left a high proportion of reads unclassified in many samples. This could be mainly due to either the absence of the organism from the database, or the sequences that Kraken cannot classify up to the level of genus, for instance when analyzing organisms with high genetic diversity.
Indeed, when using the NCBI blastn to search a random subset of unclassified reads in the non-redundant database (nr), we observed three main patterns: reads that either did not produce significant matches with any organism, or came from eukaryotes not present in our Kraken database; reads that produced partial alignments with many different taxa; and reads that produced good alignments, even with the target organism, but having alignment identities below 90\%, what makes Kraken unable to find exact matches of $31 \mathrm{bp}$.

\section{Implementation of a contamination-aware analysis pipeline: Mycobacterium tuberculosis as a test case}

The analysis of the bacterial dataset revealed that contaminant reads can have a major impact in bacterial diversity estimations. It is clear, however, that a unique approach might not be appropriate for all organisms and that implementations of contamination-aware pipelines must take into account the genetic particularities of each organism and rely on comprehensive validations. Following the analysis of the bacterial dataset, we implemented and extensively evaluated two contamination-control approaches on top of a specific analysis pipeline for $M$. tuberculosis, which is the pathogen our laboratory is focused on. We tested the Kraken-based taxonomic filter 
at species level (Mycobacterium tuberculosis complex) and a similarity filter that removes read mappings with identity and length lower than $97 \%$ and $40 \mathrm{bp}$, respectively. We tested both approaches using simulated and real sequencing runs. In first instance, we used simulated experiments to evaluate how non-MTB reads are mapped to the MTB reference genome and quantify the false positive and negative SNPs that arise consequently. We mapped simulated sequencing samples of 45 organisms to the MTB reference genome, including oral and respiratory microbiota, clinically common nontuberculous mycobacteria, and human reads. As expected, conserved genes like the $16 \mathrm{~S}, r p o B$, or the tRNAs constitute hotspots where contaminant sequences are frequently mapped. However, non-MTB alignments are not only produced in these regions but across the reference genome (Fig. 4a). This is dependent on the phylogenetic relationship of the contaminant organism to the one being studied. Non-tuberculous mycobacteria represent the best example of this, as their read mappings can produce high sequencing depths along the MTB reference genome. Human reads, which are a frequent concern in clinical studies, did not produce any alignments at all.

Next, we evaluated the performance of the taxonomic filter and the similarity filter using in silico contaminated samples. Although both approaches reduced the number of non-MTB mappings, the taxonomic filter showed the best performance, eliminating all non-MTB alignments with the only exception of a proportion of Mycobacterium avium reads. Accordingly, the number of false positive vSNPs due to contaminants was reduced with both methods, but in the case of the taxonomic filter, almost all erroneous SNP calls were eliminated (Fig. 4b). Only contamination with $M$. avium, a closely related bacteria, compromised its performance. Nonetheless, the errors observed were notably lower than when only using a mapping quality threshold (60 in this work). For example, when a $5 \%$ of $M$. avium was present, the 3325 false positive vSNPs and 51 false negative fSNPs identified were reduced to 24 and 9, respectively, after applying the taxonomic filter. The few false negative fSNPs observed in Fig. 4b which are systematic between all methods were due to some positions next to hard-tomap regions that do not pass the coverage cutoffs required to call a fSNP in contaminated samples.

Remarkably, even a $5 \%$ of contaminating reads can introduce a large number of false positive vSNPs. As expected, the erroneous calls produced by such small contamination fall mainly in conserved regions. However, in agreement with the results shown in Fig. 4a, spurious SNPs can be called across the genome (Additional file 8: Figure S2). Importantly, it is precisely because many of the contaminant alignments are produced in conserved genes that we predicted false antibiotic resistances, including well-known mutations to first line drugs in MTB treatment (Additional file 9: Table S7).

We also evaluated whether these filters systematically remove sequencing reads from particular genomic regions leading to biases produced by the methodology itself. To do so, we analyzed the mean sequencing depth obtained across the genome, before and after applying the filters, for all the samples of the MTB dataset that have less than $1 \%$ of contamination (984 samples; $78 \%$ of the samples analyzed). Importantly, we observed the taxonomic filter to systematically remove sequencing reads coming from the $16 \mathrm{~S}$ gene due to the inability of Kraken to classify many reads coming from this gene up to the level of species. However, for the rest of the genome, it showed an excellent performance, with virtually no differences in depth, even for conserved regions like the $r p o B$ gene (Additional file 10: Table S8). On the contrary, the similarity filter produced a systematic decrease in depth across the genome. In the $97 \%$ of the genome, the sequencing depth was reduced more than $1 \times$, with several regions showing larger decreases (Additional file 11: Table S9).

\section{Impact of contamination in clinical WGS samples of Mycobacterium tuberculosis}

After evaluating the performance of the taxonomic and similarity filters, we used them to remove contaminants in a dataset comprising 1553 MTB WGS samples from 8 different studies. As done for the bacterial dataset, we only analyzed samples with at least $50 \%$ of reads classified as Mycobacterium tuberculosis complex and 40x of median sequencing depth $(20 \times$ for direct sequencing from clinical specimens) to discard heavily contaminated samples (1267 samples, $81.6 \%$ of the MTB dataset).

Given that the taxonomic filter showed to be extremely conservative with all genomic positions except the $16 \mathrm{~S}$ gene, we discarded from the following analyses any SNP called in this region ( $r r s, r r l, r r f)$. Therefore, the differences observed in variant analysis when applying this filter can be attributed to noise introduced by contamination. In accordance, we expected no differences in variant calling in samples not affected by contaminants. When analyzing real WGS MTB samples with the taxonomic filter, we observed no variant change for 788 samples (62\% of the samples analyzed). Importantly, this agreement was true for samples with low-level contamination (less than 1\%) but also for samples with higher number of contaminant reads (up to $31 \%$ ), probably from organisms genetically distant to MTB. Overall, the numbers of SNPs either removed or recovered after applying the taxonomic filter were independent of the level of contamination of a sample (Pearson's correlation coefficient $=0.03$ ). Altogether, these results strongly 


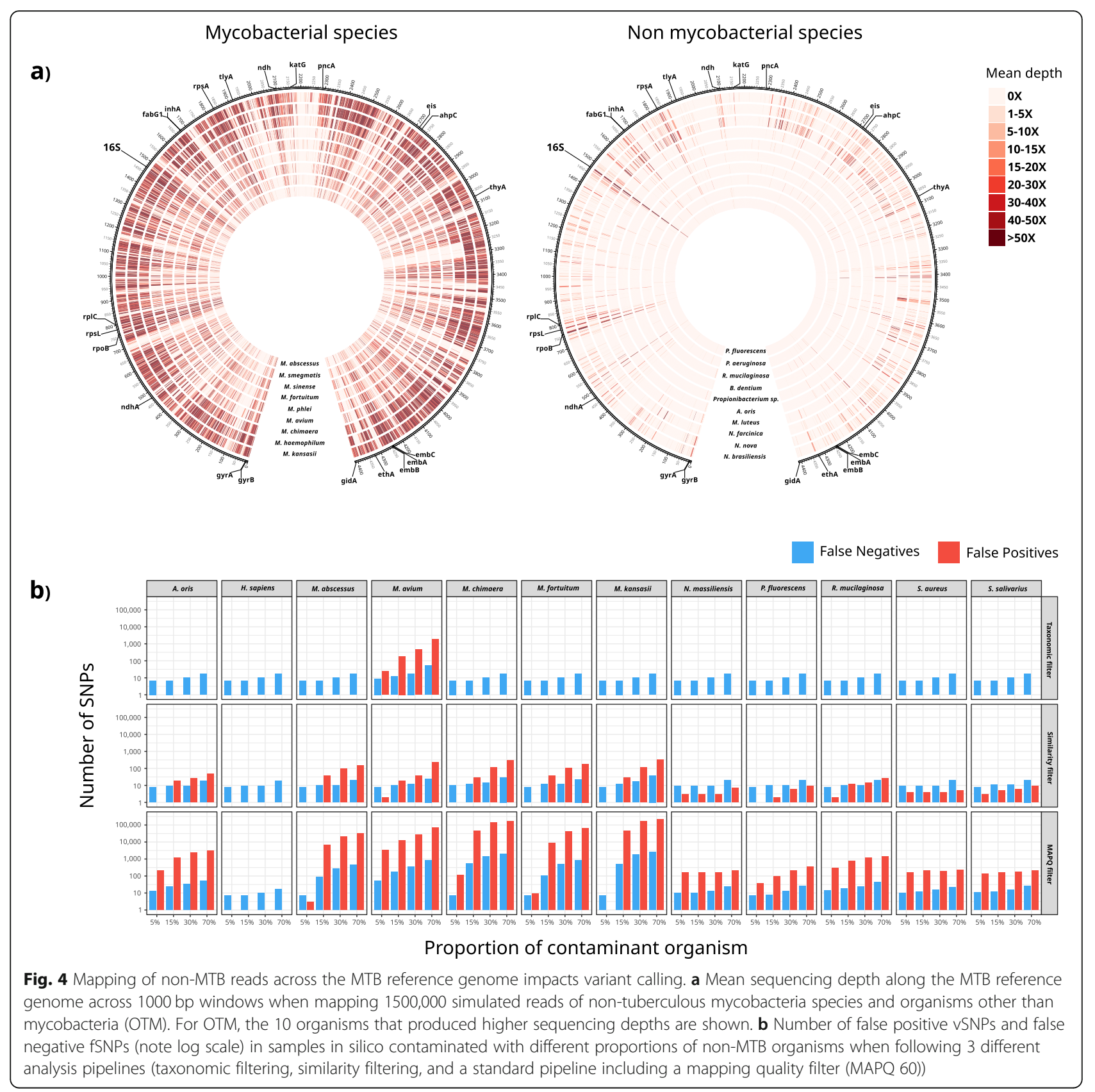

suggest that the changes observed in variant analysis after applying the taxonomic filter can be attributed to noise introduced by contaminants rather than a methodological bias. On the contrary, the similarity filter always removes variant positions even for the 984 samples with $99 \%$ of MTB. This is in agreement with the higher rate of false negatives observed in the in silico experiments.

Mapped contaminant reads introduce new variants that alter the allele frequencies. After applying the taxonomic filter, we observed a mean change of $42 \%$ allele frequency $\quad($ median $=41 \% ; \mathrm{IQR}=36 \%$ ). As shown in Fig. 5, the main consequence of these alterations is the introduction of many false positive vSNPs, even for samples with contamination levels as low as $5 \%$. However, altering allele frequencies can also lead to call false negative vSNPs, and false positive and negative fSNPs. Among the $38 \%$ of samples for which at least 1 change was observed, the taxonomic filter removed on average 761.7 vSNPs (median $=18$ ) and 4 fSNPs $($ median $=1)$, and recovered 1.7 vSNPs (median $=1$ ) and 5.9 fSNPs $($ median $=2)$. On average, the total number of polymorphic positions within each study was reduced by $0.4 \%$ for fSNPs (range 0-2\%) and $43 \%$ for vSNPs (range 3-95\%) (Fig. 3, Additional file 5: Table S5). Applying the 


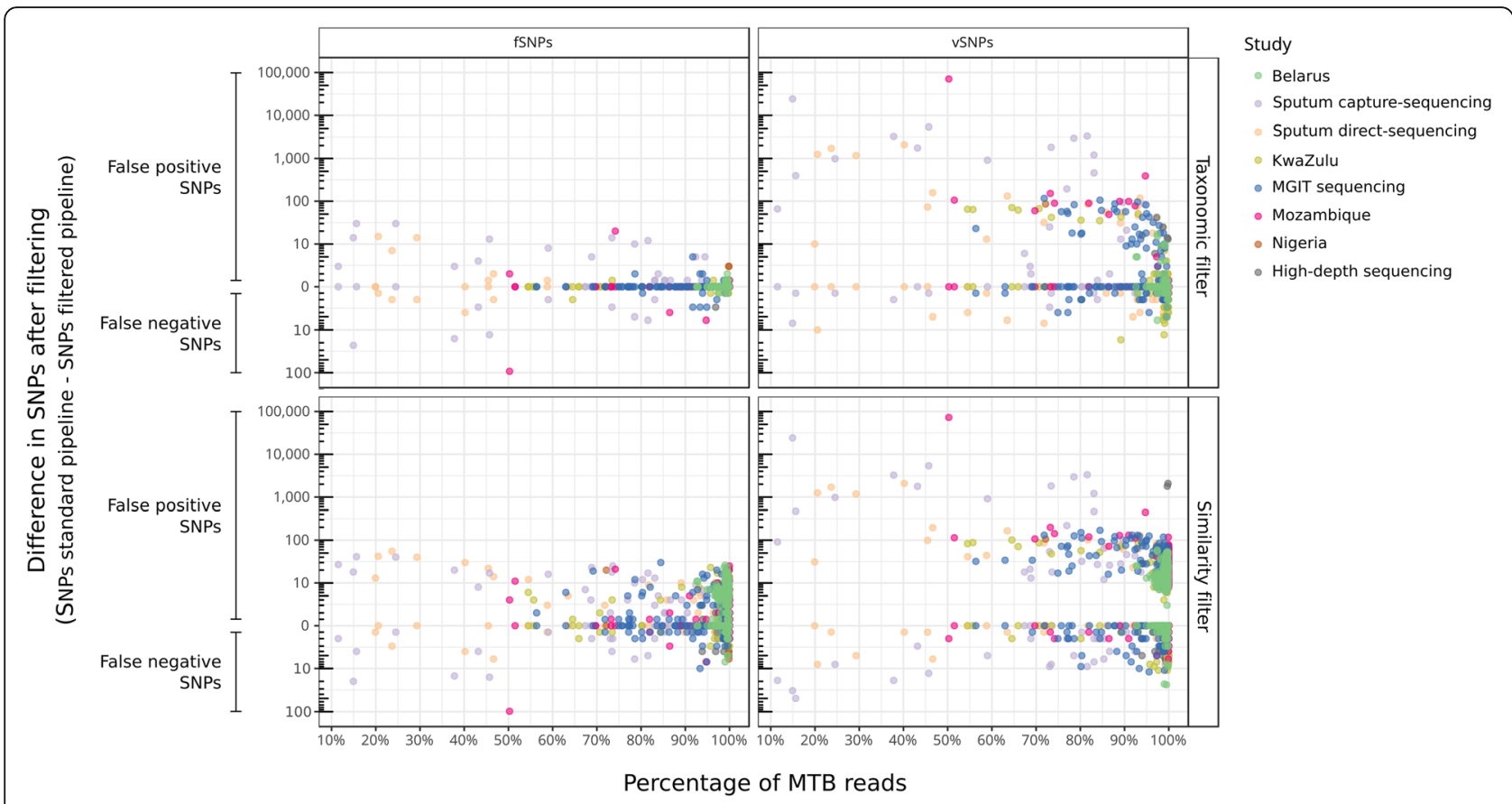

Fig. 5 Differences in SNP calling in samples of the MTB dataset between a standard pipeline and the two contamination-control methodologies tested

similarity filter removed on average 129.1 vSNPs (median $=20)$ and $6.1 \mathrm{fSNPs}($ median $=5)$ and recovered 2.6 vSNPs $($ median $=2)$ and 2.3 fSNPs $($ median $=2)$.

Sequencing directly from clinical specimens is subject to greater alterations in variant analysis (Fig. 5) since this strategy usually yields highly contaminated samples and limited sequencing depth. In these cases, the SNP frequencies are more sensitive to contaminant reads since only few reads can be responsible for a shift in the frequencies that make a position to fall below or above the required thresholds to call a variant (Additional file 7: Figure S1). However, a high sequencing depth does not guarantee an analysis safe of errors either. This effect can be observed in the high-depth sequencing study, a work based on low-frequency variant analysis from samples with more than $1000 \times$ sequencing depth. In this study, 7 samples out of 63 showed changes in the SNP analysis after applying the taxonomic filter. On average, 16.9 false positive vSNPs were removed (ranging from 2 to 42 vSNPs), and for 1 sample, 3 false negative fSNPs and 2 vSNPs were recovered. Remarkably, no strong contamination was detected for these samples (with MTB ranging from 96.86 to $99.84 \%$ ). For instance, in a sample with as much as $99.84 \%$ of MTB, the taxonomic filter removed 13 false positive vSNPs in 12 different genes across the genome.

\section{Discussion}

In this work, we analyze more than 4000 WGS samples from 14 different pathogenic bacterial species to evaluate the extent and impact of contamination in bacterial WGS studies. We show that presence of sequencing reads from contaminating organisms is frequent, even when sequencing is performed from pure culture isolates (Fig. 1). Beyond inappropriate laboratory practices, there are several potential sources of contamination which depend on different factors such as the type of sample processed and its origin, or the protocols followed for culture, DNA extraction, and sequencing. For instance, Salter et al. demonstrated that contaminating DNA in laboratory reagents can critically impact microbiome analysis from low-biomass samples [19]. Culture-free sequencing approaches for unculturable or slow-growing pathogens, such as T. pallidum or MTB, entail the presence of high amounts of contaminating DNA from the host organism. Other sources unrelated to sample handling are also possible. For example, the $S$. aureus samples supposed to be MTB from the Nigeria study are most likely an error during data submission to the genomic repository. Regardless of the source of contamination, the shared consequence is the presence of non-target reads in the sequencing files that might impact the results of genomic analysis.

We evaluated such an impact and demonstrate that contaminant reads suppose a pitfall in re-sequencing pipelines, since they are unexpectedly frequent and can have major implications in variant analysis, which is the foundation of many genomic analyses. As expected, contamination is a major issue when sequencing DNA that 
has not been extracted from pure cultures or single colonies, as is often the case for clinical specimens. However, we show that experiments sequencing from pure cultures are not necessarily free of contamination, and that using standard mapping quality parameters is not enough to deal with contaminant reads. Therefore, bioinformatic pipelines assuming that all the reads successfully mapped are from the target organism might lead to a biased variant analysis. We show that the errors introduced by contamination are very variable among different studies, (Table 2; Fig. 3; Fig. 5), which differ not only on the organism being sequenced but also on the sampling source and laboratory protocols. For example, in the T. pallidum study, where samples are heavily contaminated, very few differences are observed in the variant analysis. This stems from the fact that most of the contamination in this study comes from human reads, unlikely to map to the T. pallidum genome. On the contrary, for the L. pneumophila dataset, a sample with 96.27\% of Legionella had 79 vSNPs and 5 fSNPs removed, and 17 fSNPs recovered after filtering a 3\% of unclassified reads. According to the NCBI blast, a fraction of those reads was from Legionella spiritensis. The downstream relevance, however, is not directly proportional to the absolute number of erroneous SNPs and frequencies, but to what that errors mean for each organism. For example, for organisms with low genetic diversities, like in the case of MTB, a change in few fSNPs can have major implications in epidemiology studies since transmission cutoffs vary between 5 and 12 fSNPs [20]. This is also true when predicting drug resistance, particularly considering that many drug resistanceassociated genes are conserved among bacteria and hence more prone to recruit contaminant mappings. Likewise, the higher impact observed for vSNPs, both in terms of absolute numbers and frequencies, can have large implications in those applications based on the analysis of the allele frequency spectrum, for example, when studying complex traits in bacterial populations. For instance, vSNPs are analyzed to determine heteroresistance to antibiotics [21], within host diversity of pathogens [22], host adaptation of bacteria [23], and even to delineate between patient transmission of pathogens [24]. While not specifically tested in our analysis, our results also have obvious implications in other applications which highly depend on the variation detected (e.g., cgMSLT typing) or when contaminant reads are incorporated in de novo assemblies [18].

The main limitation of our study is that we have based our estimations on the taxonomic classifications of Kraken. However, taxonomic classifiers are known to misclassify a proportion of reads that are thus incorrectly identified as contaminants. We took into account several considerations to control for the potential biases in our analysis. Whereas Kraken is computationally expensive, its performance has been demonstrated in several studies to rank among the best up to date [25-27]. Secondly, since distinguishing between closely related species may be difficult, to be conservative, we performed the taxonomic filter at the genus level instead of species. Additionally, we estimated the error introduced by Kraken in our own setting, particularly regarding unclassified and misclassified reads, and showed that the error rate was very low (Additional file 1: Table S1; Additional file 2: Table S2; Additional file 3: Table S3), in agreement with published data. Despite these measures, we might have under- or overestimated the impact of contaminant reads in some cases. For example, by removing nontarget reads at the level of genus, we might have underestimated the impact of potential contamination, given that contaminant reads from the same genus (but different species) are more likely to map to the reference genome and thus impact variant analysis. Our analysis also showed that Kraken might have overestimated the number of contaminant reads in some datasets due to, for example, exchange of genetic material between species (K. pneumoniae, S. aureus, S. enterica) or the absence of enough genetic diversity in the database.

Altogether our results show that contaminant reads in re-sequencing experiments are frequent and can greatly bias variant analysis at a genomic level. However, based on our results, it is clear that different settings will require different contamination-control strategies that take into account the genetic particularities of each organism. Whereas the taxonomic filter we propose seems to perform well in many situations, in the case of highly diverse bacteria, other approaches might be better suited. For instance, coverage information and $k$-mer frequencies $[28,29]$ can be used to distinguish between target and contaminant reads when these are present in significantly different proportions. Similarly, detecting crosscontamination with strains of the same species is challenging and requires specific strategies. These strategies can include detection of vSNPs at lineage-defining positions, calculation of biased allele ratios [30], or Bayesian statistical modeling [31].

Importantly, different implementations of such strategies should be extensively evaluated and validated. Here, we provide such an evaluation for the pathogen our laboratory is focused on: M. tuberculosis. In addition to the taxonomic filter, we evaluated a second contaminant filtering approach based on the similarity of the read alignments. In this case, the Kraken-based taxonomic filter clearly outperformed the similarity filter what is probably true for other organisms with representative genomes in the databases and moderate genetic diversities (Fig. 4, Fig. 5, Additional file 1: Tables S8 and S9, and Figure S1). 
The analyses for MTB reveal a large number of variants introduced by contaminants with downstream consequences when calling vSNPs and fSNPs as well as the wild type. Remarkably, we show that contamination can introduce substantial errors in samples that could be considered "pure" or with high sequencing depths, implying that contamination-aware pipelines will be needed in any circumstance.

Contamination has been recognized as a major source of error in genome assemblies and other fields like metagenomics $[16,19]$. However, the role of contamination in re-sequencing pipelines is usually neglected. Whereas some groups are already aware of this issue, most bacterial re-sequencing pipelines are still lacking contaminationcontrol strategies or, if any, these are rarely detailed in published works. Based on our findings, we call for the inclusion of contamination control as a basic quality parameter and the use of validated contamination-aware pipelines in any bacterial WGS study. These analyses pipelines should be capable of, at least, reporting the contaminated samples and their contaminants to be later interpreted by the researcher. Ideally, they should be able to produce accurate results regardless of the extent of contamination of a sample. Pipelines capable of accurately analyzing contaminated WGS data will soon become essential, since the improvement of laboratory protocols allows the sequencing of an increasing number of bacterial species directly from clinical specimens [32, 33]. In this work, we provide a highly accurate contamination-aware pipeline for MTB WGS analysis that will be extremely helpful in the upcoming studies and clinical applications sequencing MTB directly from respiratory samples.

\section{Material and methods}

\section{Datasets analyzed from bacterial WGS studies}

In order to detect contamination through different studies and evaluate its impact in bacterial WGS experiments, we analyzed WGS runs from 20 different studies. We considered studies that have been published recently and for which Illumina sequencing reads were already available. The datasets comprised 8 MTB studies and 12 studies of other 13 relevant pathogenic species. Nineteen of these datasets were publicly available beforehand [34-52]. To include a dataset generated in our laboratory, we sequenced 138 MTB samples from Mozambique in the Illumina MiSeq platform. A total of 4194 Illumina runs were analyzed, comprising 1553 MTB samples (MTB dataset) and 2641 samples from the rest of organisms (bacterial dataset) (Table 1).

\section{Whole-genome sequencing of MTB samples from Mozambique}

DNA extractions were performed in heat-inactivated samples of MTB Löwenstein-Jensen cultures with an automated DNA extraction platform (NucliSENS EasyMag;
bioMérieux). Sequencing libraries were prepared with Nextera XT DNA Library Preparation Kit v3 (Illumina, San Diego, CA) following the manufacturer's instructions. Whole-genome sequencing was performed on the Illumina MiSeq instrument with 2X300bp paired-end reads.

\section{Contamination assessment using Kraken}

In order to assess contamination in each dataset, sequencing reads were taxonomically classified using Kraken [53] with a custom database comprising all sequences of bacteria, archaea, viruses, protozoa, plasmids, and fungi in RefSeq (release 78), plus the human genome (GRCh38, Ensembl release 81). Kraken classifications and Kraken database setup were performed with default parameters. Bracken [54] was used to estimate the number of misclassified reads that could be reassigned to the target organism.

\section{Analysis pipeline}

To analyze WGS data, we used a general analysis pipeline for read mapping and variant calling. In summary, bases with an average quality below 25 in a 20-bp window were trimmed and reads shorter than $50 \mathrm{bp}$ were filtered. Sequences were then mapped to the reference genome of each organism using bwa-mem [55]. We used as reference genomes those used by the authors in their respective manuscripts when specified and otherwise the representative genome of RefSeq (Additional file 12: Table S10). For MTB samples, we used the genome of the inferred most recent common ancestor of the Mycobacterium tuberculosis complex. Alignments with mapping qualities (MAPQ) below 60 were removed. Variants were then called and filtered using two different set of parameters to call fixed SNPs (fSNPs) and variable SNPs (vSNPs). The cutoffs to call fSNPs were minimum depth of 20 reads, with the variant observed in at least 20 reads; average base quality of $25 ; p$ value cutoff of 0.01 , observed in both strands; and minimum frequency of $90 \%$. The cutoffs to call vSNPs were minimum depth of 10 reads, with the variant observed in at least 6 reads; average base quality of $25 ; p$ value cutoff of 0.01 , observed in both strands; and minimum frequency of $10 \%$. We also removed SNPs near indels in a window of $4 \mathrm{bp}$. For MTB samples, we used an additional annotation filter to remove SNPs in repetitive and mobile regions. Additionally, to call fSNPs, we used a density filter removing SNPs within high-density regions (allowing a maximum of 3 SNPs in $10 \mathrm{bp}$ windows). This filter is commonly used in MTB WGS data since it is not expected to observe many contiguous variants given the extremely low genetic diversity of this species.

We compared this general analysis pipeline with two approaches for contamination removal. The "taxonomic 
filter" consisted of the removal of contaminant reads after the trimming step, prior to mapping. For MTB samples, we removed those reads classified by Kraken as any species other than Mycobacterium tuberculosis complex. In the case of organisms other than MTB, to be conservative, we removed the reads classified as any organism other than the target at the level of genus, keeping also those sequences classified as phages of those organisms. For MTB samples, we additionally evaluated a method consisting in a custom similarity filter. We tested several combinations that filtered the alignments based on their similarity, length, and mapping quality (Additional file 13: Figure S3). The similarity filter finally consisted in the removal of alignments with length, identity, and quality below $40 \mathrm{bp}, 97 \%$, and 60, respectively.

We only considered for analysis samples with more than the $50 \%$ of the target organism and with a median sequencing depth of at least $40 \times$. In the case of studies performing WGS directly in clinical samples (sputumcapture sequencing, sputum-direct sequencing, and Treponema studies), we analyzed those samples that had at least $20 \times$ of median coverage, since this type of sequencing is expected to sequence samples with lower coverages and high proportions of non-target reads.

\section{Generation of simulated datasets}

We used the reference genome of each organism to generate simulated sequencing samples using ART [56]. We generated paired-end sequencing data of 250 and $100 \mathrm{bp}$ using the error profiles of Illumina MiSeq (--ss MSv3) and Illumina HiSeq (-ss HS20) platforms, respectively. This allowed us to estimate the proportion of reads that cannot be classified by Kraken up to level of genus and species for each organism. The same approach was used to generate sequencing runs of different bacterial contaminants commonly observed in MTB WGS samples (see below). The command line used to generate the sample was as follows: art_illumina -ss [MSv3 | HS20] --rcount 2000000 -1 250 --mflen 800 --paired --minQ 25 -s 300

\section{Evaluation of the impact of contamination and methodology validation}

We generated simulated sequencing of the MTB reference genome, the human genome (GRCh38, Ensembl release 81 ), and 44 different non-MTB bacterial species (Additional file 14: Table S11). This allowed us to perform two kinds of experiments (mapping of non-MTB reads to the MTB reference genome and analysis of mock contaminated samples) as explained further below.

In order to inspect which regions of the reference genome are susceptible of recruiting non-MTB reads, we mapped the simulated reads and then measured the mean sequencing depth across the genome in $1000 \mathrm{bp}$ windows. To assess whether false positive SNPs and drug resistance predictions are produced by these nonMTB mappings, we generated mock contaminated samples by mixing sequencing reads of the reference genome with different proportions (5\%, 15\%, 30\%, and 70\%) of other organisms corresponding to 12 common contaminants identified in the MTB dataset. Therefore, any SNP identified when analyzing these samples would be a false positive, attributable to contamination.

In addition, we mapped these mock samples to a modified version of the reference genome where we introduced random mutations each $100 \mathrm{bp}$, and all the drug resistance conferring mutations described as "high confidence" in the PhyResSE catalog [57]. Therefore, any of the introduced SNPs that were undetected when analyzing these samples would be false negatives attributable to contamination.

\section{Supplementary information}

Supplementary information accompanies this paper at https://doi.org/10. 1186/s12915-020-0748-z.

Additional file 1: Table S1. Evaluation of the performance of Kraken classifying reads at genus and species level for the reference genomes and among all samples of the studies analyzed.

Additional file 2: Table S2. Evaluation of the performance of Kraken classifying reads at genus and species level for the reference genomes and among all samples of the studies analyzed after excluding the reference genomes from the Kraken database.

Additional file 3: Table S3. Bracken estimation of the proportion of misclassified and unclassified reads that actually belong to the target organism.

Additional file 4: Table S4. Samples of the bacterial dataset with less than $50 \%$ of target organism.

Additional file 5: Table S5. Difference in the number of variant positions within a dataset between the basic and the taxonomic-filtered pipeline.

Additional file 6: Table S6. Proportion of fSNPs removed per sample in the bacterial dataset. (DOCX $6 \mathrm{~kb})$

Additional file 7: Figure S1. Effects of contaminations and taxonomic filtering in variant calling.

Additional file $\mathbf{8}$ :Figure S2. Contaminations can lead to incorrect calls across the M. tuberculosis genome.

Additional file 9: Table S7. Evaluation of false drug resistance predictions in mock contaminated samples.

Additional file 10: Table S8. Genomic regions (1000 bp windows) with a coverage decrease greater than 1X after taxonomic filtering in 984 samples of the MTB dataset with more than $99 \%$ of reads classified as MTB.

Additional file 11: Table S9. Top ten genomic regions (1000 bp windows) with greater coverage decrease after applying the similarity filter in 984 samples of the MTB dataset with more than $99 \%$ of reads classified as MTB.

Additional file 12: Table S10. Reference genomes of the bacterial dataset.

Additional file 13: Figure S3. Implementation of the similarity mapping filter.

Additional file 14: Table S11. Non-MTB species included in the simulated sequencings to evaluate the impact of contaminations in MTB WGS samples. 


\section{Authors' contributions}

IC and GAG designed the study, analyzed the data, and wrote the manuscript. SB and AGB provided the MTB samples from Mozambique. All authors read and approved the final manuscript.

\section{Funding}

This work was funded by projects of the European Research Council (ERC) (638553-TB-ACCELERATE) and Ministerio de Economía y Competitividad (Spanish Government) research grant SAF2016-77346-R (to IC), SAF201792345-EXP (to IC), and BES-2014-071066 (to GAG).

\section{Availability of data and materials}

All data generated or analyzed during this study are included in this published article, its supplementary information files, and publicly available repositories. Whole-genome sequencing data from Mozambique isolates generated in our laboratory is available at the European Nucleotide Archive under the accession PRJEB27421 [58]. The inferred most recent common ancestor genome of the Mycobacterium tuberculosis complex is available at Zenodo [59].

\section{Ethics approval and consent to participate}

Not applicable.

\section{Competing interests}

The authors declare that they have no competing interests.

\section{Author details}

'Institute of Biomedicine of Valencia, IBV-CSIC, St. Jaume Roig 11, 46010 Valencia, Spain. ${ }^{2}$ Centro de Investigaçao em Saúde de Manhiça (CISM), Bairro Cambeve, Rua 12, Distrito da Manhiça, 1929 Maputo, Mozambique. ${ }^{3}$ ISGlobal, Hospital Clínic - Universitat de Barcelona, Barcelona, Spain. ${ }^{4} \mathrm{CIBER}$ in Epidemiology and Public Health, Madrid, Spain.

\section{Received: 19 August 2019 Accepted: 11 February 2020} Published online: 02 March 2020

\section{References}

1. McAdam PR, Richardson EJ, Ross FJ. High-throughput sequencing for the study of bacterial pathogen biology. Curr Opin Microbiol. 2014;19:106-13.

2. Didelot X, Bowden R, Wilson DJ, Peto TEA, Crook DW. Transforming clinical microbiology with bacterial genome sequencing. Nat Rev Genet. 2012;13: 601-12.

3. Roach DJ, Burton JN, Lee C, Stackhouse B, Butler-Wu SM, Cookson BT, et al. Correction: A year of infection in the intensive care unit: prospective whole genome sequencing of bacterial clinical isolates reveals cryptic transmissions and novel microbiota. PLoS Genet. 2017;13:e1006724.

4. Brown AC, Christiansen MT. Whole-genome enrichment using RNA probes and sequencing of Chlamydia trachomatis directly from clinical samples. Methods Mol Biol. 1616;2017:1-22.

5. SenGupta DJ, Cummings LA, Hoogestraat DR, Butler-Wu SM, Shendure J, Cookson BT, et al. Whole-genome sequencing for high-resolution investigation of methicillin-resistant Staphylococcus aureus epidemiology and genome plasticity. J Clin Microbiol. 2014;52:2787-96.

6. Lees JA, Kendall M, Parkhill J, Colijn C, Bentley SD, Harris SR. Evaluation of phylogenetic reconstruction methods using bacterial whole genomes: a simulation based study. Wellcome Open Res. 2018;3:33.

7. Bentley SD, Parkhill J. Genomic perspectives on the evolution and spread of bacterial pathogens. Proc Biol Sci. 2015;282:20150488.

8. Falush D. Bacterial genomics: microbial GWAS coming of age. Nature microbiology. 2016;1:16059.

9. Lenski RE. Experimental evolution and the dynamics of adaptation and genome evolution in microbial populations. ISME J. 2017;11:2181.

10. Campbell F, Strang C, Ferguson N, Cori A, Jombart T. When are pathogen genome sequences informative of transmission events? PLoS Pathog. 2018; 14:e1006885.

11. Fields FR, Lee SW, McConnell MJ. Using bacterial genomes and essential genes for the development of new antibiotics. Biochem Pharmacol. 2017; 134:74.

12. Didelot $X$, Walker AS, Peto TE, Crook DW, Wilson DJ. Within-host evolution of bacterial pathogens. Nat Rev Microbiol. 2016;14:150-62.
13. Olson ND, Lund SP, Colman RE, Foster JT, Sahl JW, Schupp JM, et al. Best practices for evaluating single nucleotide variant calling methods for microbial genomics. Front Genet. 2015;6:235.

14. Wilson CG, Nowell RW, Barraclough TG. Cross-contamination explains "inter and intraspecific horizontal genetic transfers" between asexual Bdelloid rotifers. Current Biol. 2018:28:2436-44 e14.

15. Ballenghien M, Faivre N, Galtier N. Patterns of cross-contamination in a multispecies population genomic project: detection, quantification, impact, and solutions. BMC Biology. 2017;15. https://doi.org/10.1186/s12915-017-0366-6.

16. Lu J, Salzberg SL. Removing contaminants from databases of draft genomes. PLoS Comput Biol. 2018;14:e1006277.

17. Merchant S, Wood DE, Salzberg SL. Unexpected cross-species contamination in genome sequencing projects. PeerJ. 2014;2:e675.

18. Breitwieser FP, Pertea M, Zimin A, Salzberg SL. Human contamination in bacterial genomes has created thousands of spurious proteins. Genome Research. 2019. https://doi.org/10.1101/gr.245373.118.

19. Salter SJ, Cox MJ, Turek EM, Calus ST, Cookson WO, Moffatt MF, et al. Reagent and laboratory contamination can critically impact sequence-based microbiome analyses. BMC Biol. 2014;12:87.

20. Meehan CJ, Moris P, Kohl TA, Pečerska J, Akter S, Merker M, et al. The relationship between transmission time and clustering methods in Mycobacterium tuberculosis epidemiology. EBioMedicine. 2018;37:410-6.

21. Band VI, Weiss DS. Heteroresistance: a cause of unexplained antibiotic treatment failure? PLoS Pathog. 2019;15:e1007726.

22. Séraphin MN, Norman A, Rasmussen EM, Gerace AM, Chiribau CB, Rowlinson M-C, et al. Direct transmission of within-host Mycobacterium tuberculosis diversity to secondary cases can lead to variable between-host heterogeneity without de novo mutation: a genomic investigation. EBioMedicine. 2019;47:293-300.

23. Sheppard SK, Guttman DS, Fitzgerald JR. Population genomics of bacterial host adaptation. Nat Rev Genet. 2018;19:549-65.

24. Martin MA, Lee RS, Cowley LA, Gardy JL, Hanage WP. Within-host Mycobacterium tuberculosis diversity and its utility for inferences of transmission. Microb Genom. 2018;4. https://doi.org/10.1099/mgen.0.000217.

25. Mclntyre ABR, Ounit R, Afshinnekoo E, Prill RJ, Hénaff E, Alexander N, et al. Comprehensive benchmarking and ensemble approaches for metagenomic classifiers. Genome Biol. 2017;18:182.

26. Gihawi A, Rallapalli G, Hurst R, Cooper CS, Leggett RM, Brewer DS. SEPATH: benchmarking the search for pathogens in human tissue whole genome sequence data leads to template pipelines. Genome Biol. 2019;20:208.

27. Ye SH, Siddle KJ, Park DJ, Sabeti PC. Benchmarking metagenomics tools for taxonomic classification. Cell. 2019;178:779-94.

28. Crusoe MR, Alameldin HF, Awad S, Boucher E, Caldwell A, Cartwright R, et al. The khmer software package: enabling efficient nucleotide sequence analysis. F1000Res. 2015;4:900.

29. Laetsch DR, Blaxter ML. BlobTools: interrogation of genome assemblies. F1000Res. 2017;6:1287.

30. Fiévet A, Bernard V, Tenreiro H, Dehainault C, Girard E, Deshaies V, et al. ART-DeCo: easy tool for detection and characterization of crosscontamination of DNA samples in diagnostic next-generation sequencing analysis. Eur J Hum Genet. 2019;27:792-800.

31. Sankar A, Malone B, Bayliss SC, Pascoe B, Méric G, Hitchings MD, et al. Bayesian identification of bacterial strains from sequencing data. Microb Genom. 2016;2:e000075.

32. Doyle RM, Burgess C, Williams R, Gorton R, Booth H, Brown J, et al. Direct whole-genome sequencing of sputum accurately identifies drug-resistant Mycobacterium tuberculosis faster than MGIT culture sequencing. J Clin Microbiol. 2018;56. https://doi.org/10.1128/JCM.00666-18.

33. Bachmann NL, Sullivan MJ, Jelocnik M, Myers GSA, Timms P, Polkinghorne A. Culture-independent genome sequencing of clinical samples reveals an unexpected heterogeneity of infections by Chlamydia pecorum. J Clin Microbiol. 2015;53:1573-81.

34. Brown AC, Bryant JM, Einer-Jensen K, Holdstock J, Houniet DT, Chan JZM, et al. Rapid whole-genome sequencing of Mycobacterium tuberculosis isolates directly from clinical samples. J Clin Microbiol. 2015;53:2230-7.

35. Votintseva AA, Bradley P, Pankhurst $L$, del Ojo EC, Loose M, Nilgiriwala $K$, et al. Same-day diagnostic and surveillance data for tuberculosis via whole genome sequencing of direct respiratory samples. 2016. https://doi.org/10. 1101/094789.

36. Pankhurst L, del Ojo EC, Votintseva AA, Walker TM, Cole K, Davies J, et al. Rapid, comprehensive, and affordable mycobacterial diagnosis with whole- 
genome sequencing: a prospective study. Lancet Respiratory Med. 2016;4: 49-58.

37. Cohen KA, Abeel T, Manson McGuire A, Desjardins CA, Munsamy V, Shea TP, et al. Evolution of extensively drug-resistant tuberculosis over four decades: whole genome sequencing and dating analysis of Mycobacterium tuberculosis isolates from KwaZulu-Natal. PLoS Med. 2015;12:e1001880.

38. Wollenberg KR, Desjardins CA, Zalutskaya A, Slodovnikova V, Oler AJ, Quiñones $\mathrm{M}$, et al. Whole-genome sequencing of Mycobacterium tuberculosis provides insight into the evolution and genetic composition of drug-resistant tuberculosis in Belarus. J Clin Microbiol. 2017;55:457-69.

39. Senghore M, Otu J, Witney A, Gehre F, Doughty EL, Kay GL, et al. Wholegenome sequencing illuminates the evolution and spread of multidrugresistant tuberculosis in Southwest Nigeria. PLoS One. 2017;12:e0184510

40. Trauner A, Liu Q, Via LE, Liu X, Ruan X, Liang L, et al. The within-host population dynamics of Mycobacterium tuberculosis vary with treatment efficacy. Genome Biol. 2017;18:71.

41. Willems S, Kampmeier S, Bletz S, Kossow A, Köck R, Kipp F, et al. Wholegenome sequencing elucidates epidemiology of nosocomial clusters of Acinetobacter baumannii. J Clin Microbiol. 2016;54:2391-4.

42. Stone NE, Sidak-Loftis LC, Sahl JW, Vazquez AJ, Wiggins KB, Gillece JD, et al. More than $50 \%$ of Clostridium difficile isolates from pet dogs in Flagstaff, USA. Carry Toxigenic Genotypes. PLoS One. 2016;11:e0164504.

43. Tyson GH, Sabo JL, Rice-Trujillo C, Hernandez J, McDermott PF. Wholegenome sequencing based characterization of antimicrobial resistance in Enterococcus. Pathog Dis. 2018;76. https://doi.org/10.1093/femspd/fty018.

44. Holt KE, Wertheim H, Zadoks RN, Baker S, Whitehouse CA, Dance D, et al. Genomic analysis of diversity, population structure, virulence, and antimicrobial resistance in Klebsiella pneumoniae, an urgent threat to public health. Proc Natl Acad Sci U S A. 2015;112:E3574-81.

45. Timms VJ, Rockett R, Bachmann NL, Martinez E, Wang Q-A, Chen SC, et al. Genome sequencing links persistent outbreak of Legionellosis in Sydney (New South Wales, Australia) to an emerging clone of Legionella pneumophila sequence type 211. Appl Environ Microbiol. 2017;84. https:// doi.org/10.1128/aem.02020-17.

46. Halbedel S, Prager R, Fuchs S, Trost E, Werner G, Flieger A. Whole-genome sequencing of recent Listeria monocytogenes isolates from Germany reveals population structure and disease clusters. J Clin Microbiol. 2018;56. https:// doi.org/10.1128/JCM.00119-18.

47. Yahara K, Nakayama S-I, Shimuta K, Lee K-I, Morita M, Kawahata T, et al. Genomic surveillance of Neisseria gonorrhoeae to investigate the distribution and evolution of antimicrobial-resistance determinants and lineages. Microb Genom. 2018;4. https://doi.org/10.1099/mgen.0.000205.

48. Marvig RL, Sommer LM, Molin S, Johansen HK. Convergent evolution and adaptation of Pseudomonas aeruginosa within patients with cystic fibrosis. Nat Genet. 2015:47:57-64.

49. Gymoese P, Sørensen G, Litrup E, Olsen JE, Nielsen EM, Torpdahl M. Investigation of outbreaks of Salmonella enterica serovar typhimurium and its monophasic variants using whole-genome sequencing, denmark. Emerg Infect Dis. 2017;23:1631-9.

50. Aanensen DM, Feil EJ, Holden MTG, Dordel J, Yeats CA, Fedosejev A, et al. Whole-genome sequencing for routine pathogen surveillance in public health: a population snapshot of invasive Staphylococcus aureus in Europe. MBio. 2016;7. https://doi.org/10.1128/mBio.00444-16.

51. Pinto M, Borges V, Antelo M, Pinheiro M, Nunes A, Azevedo J, et al. Genome-scale analysis of the non-cultivable Treponema pallidum reveals extensive within-patient genetic variation. Nat Microbiol. 2016;2:16190.

52. Greig DR, Schaefer U, Octavia S, Hunter E, Chattaway MA, Dallman TJ, et al. Evaluation of whole-genome sequencing for identification and typing of Vibrio cholerae. J Clin Microbiol. 2018;56. https://doi.org/10.1128/JCM.00831-18.

53. Wood DE, Salzberg SL. Kraken: ultrafast metagenomic sequence classification using exact alignments. Genome Biol. 2014;15:R46.

54. Lu J, Breitwieser FP, Thielen P, Salzberg SL. Bracken: estimating species abundance in metagenomics data. 2017. https://doi.org/10.7717/peerj-cs.104.

55. Li H, Durbin R. Fast and accurate short read alignment with BurrowsWheeler transform. Bioinformatics. 2009;25:1754-60.

56. Huang W, Li L, Myers JR, Marth GT. ART: a next-generation sequencing read simulator. Bioinformatics. 2012;28:593-4.

57. Feuerriegel S, Schleusener V, Beckert P, Kohl TA, Miotto P, Cirillo DM, et al. PhyResSE: a web tool delineating Mycobacterium tuberculosis antibiotic resistance and lineage from whole-genome sequencing data. J Clin Microbiol. 2015;53:1908-14.
58. Goig GA, Blanco S, Garcia-Basteiro AL, Comas I. Whole genome sequencing of MTB isolates from Moçambique. Eur Nucleotide Archive. PRJEB27421. https:/www.ebi.ac.uk/ena/data/view/PRJEB27421.

59. Comas I. Genome of the inferred most recent common ancestor of the Mycobacterium tuberculosis complex. Zenodo. https://doi.org/10.5281/ zenodo.3497110.

\section{Publisher's Note}

Springer Nature remains neutral with regard to jurisdictional claims in published maps and institutional affiliations.
Ready to submit your research? Choose BMC and benefit from:

- fast, convenient online submission

- thorough peer review by experienced researchers in your field

- rapid publication on acceptance

- support for research data, including large and complex data types

- gold Open Access which fosters wider collaboration and increased citations

- maximum visibility for your research: over $100 \mathrm{M}$ website views per year

At BMC, research is always in progress.

Learn more biomedcentral.com/submissions 